\title{
Eliminating Trachoma by 2020: Assessing Progress in Nigeria
}

\author{
Mustafa A. Nasir ${ }^{1}$, Fayez Elsawy ${ }^{1}$, Abdulaziz Omar ${ }^{1}$, Shah O. Haque ${ }^{1}$, Rans Nadir ${ }^{2}$ \\ 1. Medicine, Manchester University, Manchester, GBR 2. Medicine, Imperial College London, London, GBR
}

Corresponding author: Mustafa A. Nasir, mustafa.nasir@student.manchester.ac.uk

\begin{abstract}
Trachoma is a neglected tropical disease that causes an eye infection which can lead to blindness if left untreated. In 1998, the World Health Organisation (WHO) launched a new goal to eradicate trachoma by 2020. Over the years, in partnership with the WHO, an effective strategy plan was devised to help tackle and control the disease. This involved surgery for trichiasis, antibiotic treatment, facial cleanliness, and environmental improvement (SAFE). Consequently, the number of people affected by trachoma has significantly decreased in recent times. Despite this, trachoma remains a major public health concern in 44 countries worldwide, including Nigeria. Although improvements have been seen throughout Nigeria, the disjointed application of the SAFE strategy has delayed progress compared to other countries. Providing quality treatment to those with trachoma, in addition to improving preventative measures are challenges faced throughout the country. However, a multi-pronged approach emulating the methods of other countries is recommended to achieve trachoma elimination. This review aims to evaluate the progress and challenges faced in Nigeria with regards to eliminating trachoma.
\end{abstract}

Categories: Infectious Disease, Public Health, Epidemiology/Public Health

Keywords: trachoma, get2020, nigeria, safe

\section{Introduction And Background}

Trachoma is one of the major causes of preventable loss of vision globally with an estimated 1.9 million people blinded or visually impaired [1]. Ocular infection with Chlamydia trachomatis initially causes inflammation of the conjunctiva. Repeated exposure to the bacterium without adequate treatment causes the upper eyelid to turn inwards which is known as trachomatous trichiasis. Ultimately, this results in scarring of the eye leading to the irreversible eye pathology associated with trachoma [2]. The main form of disease transmission occurs by direct contact between individuals who spread infective droplets from the eyes and nose, but the disease can also spread by fomites and flies. For this reason, trachoma tends to manifest among young children who lack facial hygiene and mothers who have high contact hours with their offspring [3].

Received 07/13/2020

Review began 07/16/2020 Review ended 07/18/2020 Published 07/29/2020

() Copyright 2020 Nasir et al. This is an open access article distributed under the terms of the Creative Commons Attribution License CC-BY 4.0., which permits unrestricted use, distribution, and reproduction in any medium, provided the original author and source are credited.
Trachoma falls within the category of neglected tropical diseases (NTDs). NTDs are a group of communicable diseases that are described as infections that have a high prevalence among people in poverty. Indeed, the disease burden of trachoma prevails in the tropical and sub-tropical regions of Africa [4]. These areas often consist of lower-income countries where there is poor access to clean water, overpopulated regions, and a history of inadequate funding of eradication programmes.

In 1998, the World Health Organisation (WHO) launched a new goal known as 'Global Elimination of blinding Trachoma by 2020' (GET2020). Alongside this goal, the WHO also devised a strategy to help tackle and control the disease; this involved surgery for trichiasis, antibiotic treatment, facial cleanliness, and environmental improvement (SAFE) plan to combat trachoma [5].

Establishing GET2020 has set the precedence for countries to take action against trachoma. Consequently, the incidence of trachoma has decreased globally with it being eradicated as a public health concern in some countries such as Cambodia and Ghana [1]. Nonetheless, the deadline for this objective has now arrived but trachoma remains a major concern in over 40 countries, including Nigeria.

Following the formation of GET2020, Nigeria quickly became a signatory of the programme and announced aims to improve the availability of water, sanitation, and health (WASH). Subsequently, they developed many ambitious initiatives such as the 'open defecation free' campaign to improve hygiene nationally [6]. Additionally, they have worked in close collaboration with non-governmental organisations (NGOs) and pharmaceutical companies to provide healthcare to those with, and at risk of, trachoma. However, despite great strides in reducing the incidence and prevalence of trachoma, Nigeria has been unable to achieve eradication by $2020[7]$.

Trachoma remains a massive contributor to disability-adjusted life years lost in Nigeria. This review aims to evaluate many factors contributing to the inability of Nigeria to fulfil GET2020, as well as suggesting future 


\section{Review}

\section{Surgery}

The main form of surgery for trichiasis is lid surgery. This is a relatively simple procedure which is often sight-saving when done timely and correctly [8]. Since the establishment of GET2020, great effort has been put into providing surgery at a community level. To overcome the limited availability of ophthalmologists, nurses have been trained to perform lid surgery in an effort to meet the clinical demand [9]. Research has shown no significant difference in clinical outcomes between nurses and doctors [10]. These surgical efforts have seen the prevalence of trichiasis decrease significantly across several states in Nigeria [7].

However, regardless of the availability of treatment, surgery is often poorly received. In Nigeria, poor surgery uptake is mainly due to financial constraints [11]. Furthermore, as surgery is largely aimed at preventing blindness, patients often do not perceive the need for treatment early on [12]. Moreover, sterilisation of equipment has been a big cause of concern among patients; particularly with regards to blood-borne diseases such as human immunodeficiency virus [13]. To counter this obstacle, the WHO has devised a surgical checklist which promotes the need to sterilise all equipment before any surgery [14].

\section{Antibiotics}

Antibiotics, especially azithromycin and tetracycline, are the main form of treatment for active trachoma [15]. Therefore, they are an essential component of any efforts to eliminate the disease. Historically, tetracycline ointment has been the treatment of choice for active trachoma. However, the discomfort associated with daily application of tetracycline to the eye resulted in reduced patient adherence [16]. In contrast, patients found oral azithromycin, taken once weekly, to be more convenient. Indeed, patient adherence rates and greater reductions in trachoma rates have been exhibited with the use of oral azithromycin as compared to tetracycline ointment. However, azithromycin is costly which makes widespread provision difficult. To address this, the pharmaceutical firm Pfizer Inc. co-founded the International Trachoma Initiative (ITI) in 1998 - a scheme that sees collaboration between ministries of health, local government associations, and NGOs [17]. Their goal is to provide large scale azithromycin, as well as aid in the development of the other facets of the SAFE strategy.

Infection with Chlamydia trachomatis is not immediately evident. Therefore, when only people with known active trachoma are treated, a reservoir of infection remains in the community [18]. Consequently, this increases the risk of reinfection. To combat this, the WHO recommends mass drug administration (MDA). MDA is the provision of drugs to whole populations in high-prevalence areas in order to treat active trachoma. MDA is to be administered in populations with a trachoma prevalence of greater than $10 \%$ among children aged between one and nine years, with a minimum of $80 \%$ of a community receiving antibiotics [19]. In 2010, 30 local government areas were surveyed for trachoma in Nigeria and, of the 30, seven were eligible to receive a donation from the ITI to provide MDA [20]. However, Nigeria has had difficulties in mapping out the districts most affected by trachoma. This is mainly due to a lack of funding and resources. It is highly likely there are other states that are eligible to receive MDA but have not yet been surveyed [13]. Nevertheless, Nigeria received its first donation from the ITI in 2010 to provide MDA for these seven local government areas.

Nigeria adopts an antibiotic delivery model whereby two to four community drug distributors (CDDs) are selected to volunteer and execute the MDA strategy. In order to assess the distribution of azithromycin within a community, CDDs must register each member of the community who receives treatment. However, the MDA target of $80 \%$ coverage is not always met. Certain states in Nigeria have been known to have an estimated antibiotic coverage of approximately $60 \%$ [20]. Furthermore, census data and population estimates are often inaccurate or unknown; leading to a discrepancy between the reported and true coverage rates achieved [21]. These difficulties have ultimately contributed to Nigeria's shortcomings in achieving GET2020.

Considering these problems, different approaches must be undertaken to achieve timely elimination of trachoma. A proposed solution to mitigate inaccuracies in population coverage is to emulate the antibiotic delivery system used in Malawi, who achieved the GET2020 target in 2019 [22]. This approach involves registering the population prior to beginning an MDA within a village. Following registration, antibiotics are administered using a static site method whereby residents collect the antibiotics themselves. Next, the occupants are interviewed, allowing for accurate calculation of coverage rates. Following this, any people who did not receive initial treatment are offered antibiotics [23]. Emulating such proven strategies would likely lead to higher and more accurate coverage rates.

\section{Water supply}

Access to water encourages adequate hygiene and face-washing, and directly results in a reduced incidence of trachoma [24]. In Nigeria, there is a significant discrepancy in the availability of clean water between the north and south of the country which is largely due to the relatively poor rainfall in the north [25]. This is an 
In rural Nigeria, hand pump boreholes are the main sources of water. However, it is estimated that only around half of these hand pumps are functional at a given time [26]. Therefore, even fewer people in rural areas have access to clean water than initially projected. Often, this is due to a lack of adequate maintenance.

Future projects focusing on both construction and maintenance of water amenities are being implemented to improve water supply. Currently, the Federal Ministry of Water Resources, who oversee WASH activities, are in the process of launching a new project known as Village Level Operation and Maintenance (VLOM) [27]. VLOM aims to construct hand pumps which can be predominantly maintained at a village level. Furthermore, the project aims to facilitate collaboration between the government and communities in relation to building and repairing boreholes. To make this possible, local mechanics and traders will be taught key repair skills in advance. Moreover, facility porters will be employed from each community with the responsibility of maintaining water and cleaning facilities. With these strategies in place to improve water supply, a rise in hygienic practices is likely to follow, resulting in a decrease in trachoma rates [24].

\section{Environmental factors}

Eye-seeking flies, such as Musca sorbens, are responsible for a substantial proportion of trachoma transmission. These insects tend to breed in human faeces and thus prevail in areas where open defecation is common practice [28]. In Nigeria, over $20 \%$ of the population practice open defecation, with higher rates observed in the north of the country where trachoma is also more prevalent [6]. A lack of latrines is the central factor for the high rates of open defecation in these communities. To combat the issue, Nigeria has launched an 'Open Defecation Free' campaign [26]. The end goal of this campaign is to provide access to sanitation, including lavatories with soap, to all Nigerians, especially in rural areas. Studies have shown that access to basic pit latrines lead to a significant reduction in the numbers of eye-seeking flies, correlating with an overall reduction in trachoma $[29,30]$.

However, water and sanitation are heavily dependent on the behavioural norms and cultural beliefs of a community. Within Nigeria, many diseases are often assumed to be related to the spiritual world and superstitions [31]. Consequently, these beliefs have slowed the adoption of cleanliness measures in Nigeria as a means to combat water-related diseases. This has led to unhygienic routines, such as open defecation, to remain common practice even when pit latrines are available.

To tackle this, Nigeria has tried to implement community-led total sanitation (CLTS) approaches. The primary aim of CLTS is to educate a community and raise awareness about the consequences of poor hygiene [32]. CLTS has been proven to be very successful in other countries, such as India. India made significant progress towards being open defecation free following the launch of the 'Total Sanitation Campaign' [33]. This programme promoted and necessitated the need for individual latrines to be built within households. Furthermore, greater awareness was raised through media and community campaigns. Latrine use has increased by $30 \%$ in studied populations in India, largely as a result of these schemes [34]. The Nigerian government has stated that they will enter a 'technical cooperation' with the committee from India to adopt a similar approach [35]. If maintained, Nigeria may be able to replicate the results observed in India and further reduce rates of trachoma transmission.

\section{Multi-pronged approach}

There is an interdependent nature between the aforementioned approaches aimed at decreasing trachoma prevalence. Repeated single-armed strategies are ineffective in controlling and reducing trachoma rates [36]. Several studies have shown that antibiotic treatment alone is insufficient to eliminate trachoma and allows a reservoir of Chlamydia trachomatis to persist within the community. Indeed, families are significantly less likely to be re-infected by trachoma if they regularly participated in hygienic habits after antibiotic therapy [37]. Therefore, cross-sector collaboration bringing together the multiple facets of trachoma control is required to achieve eradication.

\section{Conclusions}

Nigeria has taken massive strides towards reducing trachoma prevalence. However, in contrast to a number of other countries, the WHO's GET2020 target has not been achieved. National trachoma treatment has been undertaken in Nigeria following years of neglect. However, it is clear that future interventions will have to be intensified to meet forthcoming targets. Current approaches are based on the SAFE strategy with a particular focus on improving water access and hygiene through WASH campaigns. Furthermore, great advances in drug availability have been undertaken through the ITI, which sees large scale antibiotics supplied by Pfizer Inc. Each strategy has aided in trachoma prevention in the country, but often it is a lack of cooperation between different sectors that lead to shortcomings in trachoma control. If trachoma eradication is to be achieved, further plans must see these sectors augment their strategies and work in cooperation. 


\section{Additional Information \\ Disclosures}

Conflicts of interest: In compliance with the ICMJE uniform disclosure form, all authors declare the following: Payment/services info: All authors have declared that no financial support was received from any organization for the submitted work. Financial relationships: All authors have declared that they have no financial relationships at present or within the previous three years with any organizations that might have an interest in the submitted work. Other relationships: All authors have declared that there are no other relationships or activities that could appear to have influenced the submitted work.

\section{References}

1. WHO: trachoma. (2020). Accessed: July 06, 2020: https://www.who.int/news-room/factsheets/detail/trachoma.

2. Gambhir M, Basáñez MG, Turner F, Kumaresan J, Grassly NC: Trachoma: transmission, infection, and control. Lancet Infect Dis. 2007, 7:420-427. 10.1016/S1473-3099(07)70137-8

3. Mariotti SP, Pascolini D, Rose-Nussbaumer J: Trachoma: global magnitude of a preventable cause of blindness. Br J Ophthalmol. 2009, 93:563-568. 10.1136/bjo.2008.148494

4. Molyneux DH, Savioli L, Engels D: Neglected tropical diseases: progress towards addressing the chronic pandemic. Lancet. 2017, 389:312-325. 10.1016/S0140-6736(16)30171-4

5. Kuper H, Solomon AW, Buchan J, Zondervan M, Foster A, Mabey D: A critical review of the SAFE strategy for the prevention of blinding trachoma. Lancet Infect Dis. 2003, 3:372-381. 10.1016/S1473-3099(03)00659-5

6. Making Nigeria open-defecation-free by 2025. (2016). Accessed: July 06, 2020: https://www.unicef.org/nigeria/media/1491/file/Nigeria-making-Nigeria-open-defecation-free-by2025.pdf.pdf.

7. Mpyet C, Muhammad N, Adamu MD, et al.: Impact survey results after SAFE strategy implementation in 15 local government areas of Kebbi, Sokoto and Zamfara states, Nigeria. Ophthalmic Epidemiol. 2018, 25:103114. 10.1080/09286586.2018.1481984

8. Rajak SN, Habtamu E, Weiss HA, et al.: The outcome of trachomatous trichiasis surgery in Ethiopia: risk factors for recurrence. PLoS Negl Trop Dis. 2013, 7:e2392. 10.1371/journal.pntd.0002392

9. Ramyil A, Bascaran C, Bunce C, Alada J, Wade P, Mpyet C: Outcome of trachoma lid surgeries in Jigawa state, Nigeria. Cogent Med. 2016, 3:1233683. 10.1080/2331205X.2016.1233683

10. Alemayehu W, Melese M, Bejiga A, Worku A, Kebede W, Fantaye D: Surgery for trichiasis by ophthalmologists versus integrated eye care workers: a randomized trial. Ophthalmology. 2004, 111:578584. 10.1016/j.ophtha.2003.06.030

11. Rabiu MM, Abiose A: Magnitude of trachoma and barriers to uptake of lid surgery in a rural community of northern Nigeria. Ophthalmic Epidemiol. 2001, 8:181-190. 10.1076/opep.8.2.181.4167

12. Bowman RJ: Trichiasis surgery. Community Eye Health. 1999, 12:53-54.

13. Rabiu M, Muhammed N, Isiyaku S: Challenges of trachoma control: an assessment of the situation in northern Nigeria. Middle East Afr J Ophthalmol. 2011, 18:115-121. 10.4103/0974-9233.80699

14. Fast O, Fast C, Fast D, Veltjens S, Salami Z, White MC: Limited sterile processing capabilities for safe surgery in low-income and middle-income countries: experience in the Republic of Congo, Madagascar and Benin. BMJ Glob Health. 2017, 2:e000428. 10.1136/bmjgh-2017-000428

15. King JD, Teferi T, Cromwell EA, et al.: Prevalence of trachoma at sub-district level in Ethiopia: determining when to stop mass azithromycin distribution. PLoS Negl Trop Dis. 2014, 8:e2732. 10.1371/journal.pntd.0002732

16. Schachter J, West SK, Mabey D, et al.: Azithromycin in control of trachoma . Lancet. 1999, 354:630-635. 10.1016/S0140-6736(98)12387-5

17. Kumaresan J: Can blinding trachoma be eliminated by 20/20? . Eye. 2005, 19:1067-1073. 10.1038/sj.eye.6701963

18. Webster JP, Molyneux DH, Hotez PJ, Fenwick A: The contribution of mass drug administration to global health: past, present and future. Philosophical Transactions of the Royal Society B. 2014, 369:20130434. 10.1098/rstb.2013.0434

19. Jimenez V, Gelderblom HC, Mann Flueckiger R, Emerson PM, Haddad D: Mass drug administration for trachoma: how long is not long enough?. PLoS Negl Trop Dis. 2015, 9:e0003610. 10.1371/journal.pntd.0003610

20. Cromwell EA, King JD, McPherson S, et al.: Monitoring of mass distribution interventions for trachoma in plateau state, Nigeria. PLoS Negl Trop Dis. 2013, 7:e1995. 10.1371/journal.pntd.0001995

21. Cromwell EA, Ngondi J, Gatpan G, et al.: Estimation of population coverage for antibiotic distribution for trachoma control: a comparison of methods. Int Health. 2009, 1:182-189. 10.1016/j.inhe.2009.09.002

22. 18 million Malawians saved from trachoma threat . (2019). Accessed: July 06, 2020 https://www.nyasatimes.com/8-million-malawians-saved-from-trachoma-threat/.

23. Khumbo K, Salomie B, Alvin C, Micheal M, Thomas K, Ranneck S, George M: Report of the 2015 Malawi trachoma mass drug administration (mda) coverage survey in 9 districts. Adv Ophthalmol Vis Syst. 2017, 6:37-40. 10.15406/aovs.2017.06.00167

24. Cairncross S: Trachoma and water. Community Eye Health. 1999, 12:58-59.

25. Smith JL, Sivasubramaniam S, Rabiu MM, Kyari F, Solomon AW, Gilbert C: Multilevel analysis of trachomatous trichiasis and corneal opacity in Nigeria: the role of environmental and climatic risk factors on the distribution of disease. PLoS Negl Trop Dis. 2015, 9:e0003826. 10.1371/journal.pntd.0003826

26. Promising practices In WASH: some case studies of Nigeria . (2018). Accessed: July 06, 2020: https://www.unicef.org/nigeria/sites/unicef.org.nigeria/files/2018-09/Nigeria-promising-practices-inWASH.pdf.

27. Ensuring sustainability and improving functionality of water supply facilities through VLOM in Nigeria- 


\section{Cureus}

from pilot to national strategy. (2018). Accessed: July 06, 2020

https://www.unicef.org/nigeria/media/1666/file/NIgeria-WEDC-international-conference-Khan3047.pdf\%20.pdf.

28. Emerson PM, Bailey RL, Walraven GEL, Lindsay SW: Human and other faeces as breeding media of the trachoma vector Musca sorbens. Med Vet Entomol. 2001, 15:314-320. 10.1046/j.0269-283x.2001.00318.x

29. Robinson A, Bristow J, Holl MV, et al.: Responses of the putative trachoma vector, Musca sorbens, to volatile semiochemicals from human faeces. PLoS Negl Trop Dis. 2020, 14: e0007719. 10.1371/journal.pntd.0007719

30. Emerson PM, Lindsay SW, Alexander N, et al.: Role of flies and provision of latrines in trachoma control: cluster-randomised controlled trial. Lancet. 2004, 363:1093-1098. 10.1016/S0140-6736(04)15891-1

31. Akpabio EM: Water meanings, sanitation practices and hygiene behaviours in the cultural mirror: a perspective from Nigeria. J Water Sanit Hyg Dev. 2012, 2:168-181. 10.2166/washdev.2012.073

32. Chambers R: Going to scale with community-led total sanitation: reflections on experience, issues and ways forward. IDS Pract Pap. 2009, 2009:01-50. 10.1111/j.2040-0225.2009.00001_2.x

33. Patil SR, Arnold BF, Salvatore AL, Briceno B, Ganguly S, Colford JM, Gertler PJ: The effect of India's total sanitation campaign on defecation behaviors and child health in rural Madhya Pradesh: a cluster randomized controlled trial. PLoS Med. 2014, 11:e1001709. 10.1371/journal.pmed.1001709

34. Patwa J, Pandit N: Open defecation-free India by 2019: How villages are progressing?. Indian J Community Med. 2018, 43:246-247. 10.4103/ijcm.IJCM_83_18

35. World Toilet Day: experts worried Nigeria may overtake India as number one in open defecation . (2018). Accessed: July 06, 2020: https://www.premiumtimesng.com/health/health-features/297205-world-toiletday-experts-worried-nigeria-may-overtake-in...

36. Lavett DK, Lansingh VC, Carter MJ, Eckert KA, Silva JC: Will the SAFE strategy be sufficient to eliminate trachoma by 2020? Puzzlements and possible solutions. Sci World J. 2013, 1-18. 10.1155/2013/648106

37. Pinsent A, Burton MJ, Gambhir M: Enhanced antibiotic distribution strategies and the potential impact of facial cleanliness and environmental improvements for the sustained control of trachoma: a modelling study. BMC Med. 2016, 14:71. 10.1186/s12916-016-0614-6 